\title{
On Peculiarities of Betatron Oscillations of Accelerated Electron Bunches in Capillary Waveguides
}

\author{
Mikhail Veysman (iD) \\ Joint Institute for High Temperatures (JIHT) RAS, Izhorskaya 13/19, Moscow 125412, Russia \\ Correspondence should be addressed to Mikhail Veysman; bme@ihed.ras.ru
}

Received 7 February 2020; Accepted 11 January 2021; Published 30 January 2021

Academic Editor: Dieter Hoffman

Copyright (C) 2021 Mikhail Veysman. This is an open access article distributed under the Creative Commons Attribution License, which permits unrestricted use, distribution, and reproduction in any medium, provided the original work is properly cited.

\begin{abstract}
It is shown that the dynamics of electrons accelerated in narrow capillary waveguides is significantly influenced by the parametric excitation of their betatron oscillations. On the one hand, this excitation can irreversibly spoil the emittance of an accelerated electron bunch that limits the possibilities of their practical use. On the other hand, controlled parametric excitation of betatron oscillations can be used to generate short-pulse sources of synchrotron radiation. The article analyzes the regions of parametric instabilities, their dependence on the parameters of accelerated electron bunches and guiding structures, and their influence on the dynamics of accelerated electrons. The parameters of the generated synchrotron radiation are also estimated. Measurements of the spectral parameters of synchrotron radiation can serve as a tool for diagnostics of betatron oscillations and their excitation in the case of parametric resonances.
\end{abstract}

\section{Introduction}

The concept of electron acceleration to multi-GeV energies in laser wakefield accelerators [1-3] requires the propagation of laser pulses over distances of many Rayleigh lengths. For this purpose, both widely used plasma channels and gas filled capillary waveguides [4-7] can be effectively used.

It will be shown below that laser wakefields in narrow capillary waveguides (with an inner radius of the order of twice the characteristic radius of the transverse envelope of the laser field) can be used for efficient acceleration of electron bunches provided that parametric excitation of betatron oscillations of electrons is excluded throughout the entire acceleration stage. This excitation is caused by a periodic change in the frequency of betatron oscillations due to the interference of the eigenmodes of electromagnetic fields inside the waveguide. In particular, in [8], it was shown that it is possible to correlate specific mode-mixture of the laser field inside the capillary to specific frequency tuning of the betatron spectra. The corresponding conditions on the parameters of laser pulses and capillary waveguides for parametric excitation of betatron oscillations of electrons are analyzed below.
On the contrary, parametric excitation of betatron oscillations in capillary waveguides or in plasma channels with a specially matched ratio of the characteristic channel width to the laser spot size can be used for efficient generation of synchrotron radiation in the ultraviolet or X-ray range. This can be used as an alternative to the previously proposed schemes for creating short-pulse sources of synchrotron radiation due to oscillations of the centroid of a laser pulse in a plasma channel or in a capillary waveguide [9-11] or with the help of off-axis electron beam injection into laser-plasma accelerator [12].

Recently, it was theoretically demonstrated and experimentally confirmed that another type of instability, electron-hose one, leads to a sharp increase in the X-ray flux for interaction distances exceeding the dephasing length [13]. The efficient generation of X-ray photons with energies of several $\mathrm{keV}$ using betatron oscillations of electrons accelerated in laser wakefields has been demonstrated in a number of recent experiments [13-15].

In addition, the synchrotron radiation of accelerated electrons can be used to diagnose their betatron oscillations. Betatron oscillations of electrons in guiding structures directly determine the emittance of electron bunches [16-18]. 
Therefore, synchrotron radiation monitoring can be used to control the emittance of accelerated electron bunches [19], as well as to control the quality of accelerating wake waves. To maintain a low emittance of the accelerated electron bunch, parametric excitation of betatron oscillations should be avoided during the entire acceleration stage.

\section{Equations of Motion and Modes of Electromagnetic Field}

Consider a bunch of electrons accelerated in wakefields generated by a laser pulse propagating from left to right along the $0 z$ axis of a capillary waveguide filled with plasma with a constant electron concentration $n$. The entrance face of the capillary is at the point $z=0$. The motion of electrons in cylindrically symmetric wakefields behind the laser pulse is determined by the relativistic equations of motion, which have the following form in Cartesian coordinates [20]:

$$
\begin{aligned}
\frac{\mathrm{d} P_{z}}{\mathrm{~d} \tau} & =\partial_{\xi} \phi, \\
\frac{\mathrm{d} \xi}{\mathrm{d} \tau} & =\gamma_{e}^{-1} P_{z}-1, \\
\frac{\mathrm{d} P_{x}}{\mathrm{~d} \tau} & =\left(\frac{x}{\rho}\right) \partial_{\rho} \phi, \\
\frac{\mathrm{d} P_{y}}{\mathrm{~d} \tau} & =\left(\frac{y}{\rho}\right) \partial_{\rho} \phi, \\
\frac{\mathrm{d} x}{\mathrm{~d} \tau} & =\gamma_{e}^{-1} P_{x}, \\
\frac{\mathrm{d} y}{\mathrm{~d} \tau} & =\gamma_{e}^{-1} P_{y},
\end{aligned}
$$

where $\phi=\phi(\xi, \rho, \tau)$ is the normalized on $m c^{2} / e$ wakefield potential [21]; $x=k_{p} x, y=k_{p} y, \quad k_{p}=\omega_{p} / c$, and $\omega_{p}=\sqrt{4 \pi n e^{2} / m} ; e, m$, and $c$ are electron charge, mass, and speed of light, respectively; $P_{\alpha}, \alpha=x, y, z$, are dimensionless on $m c$ components of electrons momentum; $\gamma_{e}=\sqrt{1+|\mathbf{P}|^{2}}$ is the electron gamma factor; $\xi=k_{p}(z-c t), \quad \tau=\omega_{p} t$, $\rho=k_{p} r$, and $\widetilde{\xi}=k_{0}\left(z-c t\right.$ ), and $\tilde{\tau}=\omega_{0} \tau$ (where $k_{0}=\omega_{0} / c$, $\omega_{0}=2 \pi c / \lambda_{0}$ is the laser pulse frequency, and $\lambda_{0}$ is the laser wavelength) are dimensionless coordinates which will be used hereafter.

In the considered case of propagation of cylindrically symmetric laser pulses in capillary waveguide angular harmonics $[22,23]$ of the wake potential, $\phi$ are absent and it can be determined using the following simple equation, written under the assumption of a constant electron density inside the capillary $[2,21,24]$ :

$$
\left[\frac{\partial^{2}}{\partial \xi^{2}}+1\right] \phi=\frac{\left|\mathbf{a}_{\perp}\right|^{2}}{4}
$$

where $\mathbf{a}_{\perp}=e \mathbf{E}_{\perp} /\left(m \omega_{0} c\right)$ is the dimensionless transverse (with respect to direction of propagation $0 z$ ) complex amplitude of electromagnetic field strength, slowly varying in time scale $\omega_{0}^{-1}$ and at space length $\lambda_{0}$. Solution of (5) is

$$
\phi(\xi, \rho, \tau)=\frac{1}{4} \int_{\infty}^{\xi} \sin \left[\xi-\xi^{\prime}\right]\left|\mathbf{a}_{\perp}\right|^{2}\left(\xi^{\prime}, \rho, \tau\right) \mathrm{d} \xi^{\prime} .
$$

Assuming that the maximal power $P_{0}$ of the laser pulse is much less than the critical power $P_{\mathrm{cr}}$ for the relativistic selffocusing, $P_{0} \ll P_{\mathrm{cr}}=0.017\left(\omega_{0} / \omega_{p}\right)^{2} \mathrm{TW}$, and disregarding all nonlinear process of laser field propagation inside a capillary waveguide, one can express $\left|\mathbf{a}_{\perp}\right|^{2}$ in terms of the sum of its radial eigenmodes as follows [22, 25-27]:

$$
\left|\mathbf{a}_{\perp}\right|^{2}=a_{\max }^{2}\left|\sum_{n} 4 \widetilde{\widetilde{G}}_{n}(\xi, \tau) D_{n}(\rho)\right|^{2},
$$

where $a_{\max }$ is the maximum value of the module of dimensionless complex amplitude of electromagnetic field strength.

$$
\widetilde{\mathfrak{C}}_{n}(\xi, \tau)=C_{n} F_{\|}\left(\widetilde{\xi}+\Phi_{n}(\widetilde{\tau})\right) \exp \left(-i \Phi_{n}(\widetilde{\tau})\right),
$$

where $\tilde{\xi}=\gamma_{\mathrm{ph}} \xi, \widetilde{\tau}=\gamma_{\mathrm{ph}} \tau, \gamma_{\mathrm{ph}}=\omega_{0} / \omega_{\mathrm{p}}$ is the gamma factor of the plasma wave, $F_{\|}(\widetilde{\xi})$ is the longitudinal envelop of the laser pulse before its entrance into the guiding structure, and $C_{n}$ are modes coefficients, determined by boundary conditions at the guiding structure entrance $z=0[7,24]$. The expressions for phase factors $\Phi_{n}$ and radial modes $D_{n}(r)$ in the vicinity of the guiding structure axis $0 z$ in the considered case of symmetric propagation of laser fields in capillary waveguides can be written as

$$
\begin{aligned}
D_{n} & =J_{0}\left(\frac{u_{0, n} \rho}{\left(k_{p} R\right)}\right), \\
\Phi_{n} & =\left(\frac{\tilde{\tau}}{2}\right)\left(k_{\perp n}^{2}+1-\varepsilon_{c}\right), \\
k_{\perp n} & =\frac{u_{0, n}}{\mathscr{R}}\left(1-i \frac{\mu_{w}}{\mathscr{R}}\right),
\end{aligned}
$$

where $J_{0}$ and $u_{0, n}$ are zero-order Bessel function and its $n$-th root, respectively; $\mathscr{R}=k_{0} R ; R$ is the inner radius of a capillary; $\varepsilon_{c}=1-\gamma_{\mathrm{ph}}^{-2}$ is the permittivity of a plasma inside the capillary waveguide; and factor $\mu_{w}=\left(\varepsilon_{w}+1\right) /\left(2 \sqrt{\varepsilon_{w}-1}\right)$ is dependent on capillary wall properties, described by its permittivity $\varepsilon_{w}$.

Below we assume that the transverse size $\sigma_{r}$ of the accelerated electron bunch is much smaller than the characteristic transverse scale of the wakefield determined by the driving laser spot size $r_{0}$. For this case, expanding the wakefield potential near the axis $0 z$ over power series of the value $r / r_{0}$, one can omit all terms except quadratic one $\phi(r \longrightarrow 0) \sim\left(r / r_{0}\right)^{2}$. This corresponds to a linear dependence of the radial focusing force $F_{\rho}=\partial \phi / \partial \rho$ on distance $\rho$ from capillary axis. In accordance with (3) and (4), the electron trajectory in the transverse plane $x y$ is determined in this case by the following equations: 


$$
\begin{aligned}
\frac{\mathrm{d}^{2} x}{\mathrm{~d} \tau^{2}}+\frac{\mathrm{d} \ln \gamma_{e}}{\mathrm{~d} \tau} \frac{\mathrm{d} x}{\mathrm{~d} \tau}+\Omega^{2} x & =0, \\
\Omega(\xi, \tau) & =\sqrt{\frac{\alpha(\xi, \tau)}{\gamma_{e}(\xi, \tau)}} \\
\alpha(\xi, \tau) & =-\left.\rho^{-1} \partial_{\rho} \phi\right|_{\rho=0} .
\end{aligned}
$$

The equation for $y$ coordinate coincides with equation (11) after replacement of $x$ by $y$. Equation (11) describes the betatron oscillations of electrons in the transverse plane $x y$ with the frequency $\Omega$.

In (12), it is assumed that $\alpha>0$; that is, electrons are injected and accelerated in the focusing phase of the wakefields. As is known (see, for example, [28]), near the entrance to the capillary, matched to the laser spot size, the laser and wakefields are not regular enough due to the beats of high eigenmodes. This leads here to the inevitable formation of defocusing regions. To exclude the influence of such regions on the propagation of electrons, it is necessary to inject electrons at a sufficient distance from the entrance to the capillary, at a certain length $L_{\text {inj }}$, sufficient to filter higher modes due to their damping stipulated by energy losses through the walls of the capillary. In what follows, we assume that such filtering has been done.

It can be shown [18] that, in the case of relativistic electrons moving along the $0 z$ axis with a speed close to the speed of light, it is possible to separate their longitudinal and transverse motion that greatly simplifies the analysis of their betatron oscillations.

Having solved the equations of electron motion in the laser wakefields, one can determine the normalized transverse emittance [16-18]:

$$
\begin{aligned}
\varepsilon_{n} & =\sqrt{2\left(\varepsilon_{x, n}^{2}+\varepsilon_{y, n}^{2}\right)}, \\
\varepsilon_{x, n} & =\overline{\gamma_{e}} \varepsilon_{x}, \\
\varepsilon_{x} & =\sqrt{\overline{x^{2}} \overline{x^{\prime 2}}-\overline{x x^{\prime}}},
\end{aligned}
$$

of the bunch of electrons, which determines the degree of their angular and spacial spread, important for applications. Here, $x^{\prime}=\mathrm{d} x / \mathrm{d} z=\dot{x} / \dot{z}=P_{x} / P_{z}$ for considered relativistic electrons moving along axis $0 z$; the bar above some value $X$ means ensemble averaging; $\overline{X^{2}}=N_{b}^{-1} \sum_{i}\left(X_{i}-\bar{X}\right)^{2}, \bar{X}=$ $N_{b}^{-1} \sum_{i} X_{i}$, where index $i$ refers to $i$-th particle; $N_{b}$ is the full number of particles; and $\varepsilon_{n}=2 \varepsilon_{x, n}=2 \varepsilon_{y, n}$ for considered cylindrically symmetric case.

\section{Wakefields in Capillaries and Parametric Excitations of Betatron Oscillations}

Taking in mind (6)-(10), one can write down the following expression for wakefield potential $\phi$ :

$$
\begin{aligned}
\phi(\xi, \rho, \tau) & =\int_{\infty}^{\xi} \sin \left(\xi-\xi^{\prime}\right) \frac{a_{0}^{2}\left(\xi^{\prime}, \rho, \tau\right)}{4} \breve{\kappa}_{g}\left(\xi^{\prime}, \rho, \tau\right)\left[1+\sum_{n, k>n} \breve{\mu}_{n k}\left(\xi^{\prime}, \rho, \tau\right) \cos \left(\Omega_{n k} \tau\right)\right] \mathrm{d} \xi^{\prime}, \\
\breve{\kappa}_{g}(\xi, \rho, \tau) & =\frac{\sum_{n} \widetilde{C}_{n}^{2}(\tau) F_{\| n}^{2}(\xi, \tau) D_{n}^{2}(\rho)}{\left[\sum_{n} C_{n} F_{\| n}(\xi, \tau) D_{n}(\rho)\right]^{2}} \\
\breve{\mu}_{n k}(\xi, \rho, \tau) & =\frac{2 \widetilde{C}_{n}(\tau) \widetilde{C}_{k}(\tau) F_{\| n}(\xi, \tau) F_{\| k}(\xi, \tau) D_{n}(\rho) D_{k}(\rho)}{\sum_{n} \widetilde{C}_{n}^{2}(\tau) F_{\| n}^{2}(\xi, \tau) D_{n}^{2}(\rho)}
\end{aligned}
$$

where $a_{0}$ is the nonoscillating part of the module of complex amplitude $\left|a_{\perp}\right|$,

$$
\begin{aligned}
a_{0}(\xi, \rho, \tau) & =a_{\max } \sum_{n} 2 C_{n} F_{\| n}(\xi, \tau) D_{n}(\rho) ; \\
\widetilde{C}_{n} & =C_{n} e^{-k_{n}, \tilde{\tau}}, \\
k_{n}^{\prime} & =\frac{1}{2}\left[\Re k_{\perp, n}^{2}+\gamma_{\mathrm{ph}}^{-2}\right], \\
k_{n}^{\prime} & =\frac{1}{2}\left|\Im k_{\perp, n}^{2}\right|, \\
\Omega_{n k} & =\gamma_{\mathrm{ph}}\left(k_{k}^{\prime}-k_{n}^{\prime}\right),
\end{aligned}
$$

and $k_{\perp, n}$ is given by (10):

$$
F_{\| n}(\xi, \tau)=\exp \left[\frac{-\left(\delta \xi+\tau k_{n}^{\prime}\right)^{2}}{L_{\xi}^{2}}\right]
$$

where $\delta \xi=\xi-\xi_{\mathrm{Lc}}$, with $\xi_{\mathrm{Lc}}$ as the coordinate on $\xi$ of the laser pulse center; expression (16) for $F_{\| n}$ is written, for simplicity, with assumption of Gaussian longitudinal envelop of the laser pulse and taking in mind smallness of the parameter $\mathscr{R}^{-1} \ll 1$; and $L_{\xi}=k_{p} c t_{\mathrm{FWHM}} / \sqrt{2 \ln 2}$, where $t_{\mathrm{FWHM}}$ is the full width at half of the maximum intensity of laser pulse.

In accordance with (6)-(10), beats of electromagnetic field modes inside a capillary waveguide due to phase factors $\Phi_{n}(8)$ and (10) give rise to terms $\sim \cos \left(\Omega_{n k} \tau\right)$ in (14). These terms lead to oscillations with capillary length $z$ of the maximum (on coordinate $\xi$ ) value of the wakefield potential at capillary axis. 
Assuming that the center of the electron bunch is injected in the focusing phase of the wakefield in the vicinity of the maximum of the longitudinal acceleration force, we can obtain the following expression for the potential of the wakefield seen by these electrons at the moment $\tau$ :

$$
\begin{aligned}
\phi\left(\xi_{\text {inj }}, \rho, \tau\right) & =a_{\text {max }}^{2} A \kappa_{g}\left(\xi_{\text {inj }}, \rho, \tau\right)\left[1+\sum_{n, k>n} \mu_{n k}\left(\xi_{\text {inj }}, \rho, \tau\right) \cos \left(\Omega_{n k} \tau\right)\right], \\
\kappa_{g}\left(\xi_{\text {inj }}, \rho, \tau\right) & =\sum_{n} 4 C_{n}^{2} \Gamma_{n}(\tau) D_{n}^{2}(\rho) \sin \left[\xi_{\text {inj }}+\frac{\tau}{2}\left(\frac{1}{\gamma_{\mathrm{ph}}^{2}}-\frac{1}{\gamma_{e}^{2}}+\frac{u_{0 n}^{2}}{\mathscr{R}^{2}}\right)\right], \\
\mu_{n k}\left(\xi_{\text {inj }}, \rho, \tau\right) & =\frac{8}{\kappa_{g}} C_{n} C_{k} D_{n}(\rho) D_{k}(\rho) \Gamma_{n k}(\tau) \sin \left[\xi_{\text {inj }}+\frac{\tau}{2}\left(\frac{1}{\gamma_{\mathrm{ph}}^{2}}-\frac{1}{\gamma_{e}^{2}}+\frac{u_{0 n}^{2}+u_{0 k}^{2}}{2 \mathscr{R}^{2}}\right)\right], \\
\Gamma_{n}(\tau) & =\exp \left[-2 \frac{u_{0, n}^{2}}{\mathscr{R}^{3}} \mu_{w} \gamma_{\mathrm{ph}} \tau\right], \\
\Gamma_{n k}(\tau) & =\exp \left[-\frac{u_{0, n}^{2}+u_{0, k}^{2}}{\mathscr{R}^{3}} \mu_{w} \gamma_{\mathrm{ph}} \tau-\frac{1}{2}\left(\frac{\tau \Omega_{n k}}{\gamma_{\mathrm{ph}} L_{\xi}}\right)^{2}\right],
\end{aligned}
$$

where $\xi_{\text {inj }}$ is the distance between the point of injection of the center of the electron bunch at $\tau=0$ and the point of maximum of the accelerating force (which is also the point of zero transverse force $F_{\perp}$, i.e., the point of the boundary between the focusing and defocusing phases of the wakefield potential); $\gamma_{e}$ is the gamma factor of an electron bunch, the transverse and longitudinal dimensions of which are assumed to be much smaller than the corresponding characteristic dimensions of the wakefield that makes it possible to disregard the energy spread of electrons in the bunch; and $A=\sqrt{\pi / 2} L_{\xi} e^{-L_{\xi}^{2} / 8} / 4$, its maximum value $\max A \approx 0.38$ at $L_{\xi}=2$ (resonance condition of plasma wake excitation).

From (17), one can obtain the following expression for the maximum in the $\xi$ value of the wakefield potential on the capillary axis $\phi_{m \xi}(\tau)=\max _{\xi} \phi(\xi, \rho=0, \tau)$, assuming that the first mode has the largest amplitude:

$$
\phi_{m \xi}(\tau)=4 a_{\max }^{2} A\left[\sum_{n} C_{n}^{2} \Gamma_{n}(\tau) \cos \left(\frac{\Omega_{1 n}}{\gamma_{\mathrm{ph}}} \tau\right)+2 \sum_{n, k>n} C_{n} C_{k} \Gamma_{n k}(\tau) \cos \left(\frac{\Omega_{1 n}+\Omega_{1 k}}{2 \gamma_{\mathrm{ph}}} \tau\right) \cos \left(\Omega_{n k} \tau\right)\right]
$$

where $\Omega_{11}=0$ and $\Omega_{n k>n}=\gamma_{\mathrm{ph}}\left(u_{0, k}^{2}-u_{0, n}^{2}\right) /\left(2 \mathscr{R}^{2}\right)$ in accordance with (10) and (15).

The expression (18) with $\tau=k_{p} z$, taking into account only the first two modes, well describes the main details of the oscillations of the wakefield potential due to mode beats, including amplitude, phase, and frequency (see Figure 1, where the case of a silicon capillary with a radius matched to the laser spot size (when $r_{0} / R \approx 0.61$ ) is considered). Particularly, for this case, one has from (18) with $n, k=1,2$ :

$$
\frac{\phi_{m \xi}(z)}{\phi_{m \xi}}(z=0) \approx 0.67 e^{-0.2 y}+0.023 e^{-1.07 y} \cos (1.2 y)+0.25 e^{-0.64 y-0.18 y^{2}} \cos (0.6 y) \cos (96 y), \quad y \equiv \frac{z}{L_{\mathrm{ph}}}
$$

where $L_{\mathrm{ph}}=\lambda_{0} \gamma_{\mathrm{ph}}^{3}$ is the dephasing length [29]. Nevertheless, the finer structure of oscillations of the wakefield amplitude, caused by higher modes, can be essential, as will be shown below, as long as it can leads to parametric excitation of betatron oscillations of electrons propagating in a capillary. This structure, due to the first 6 modes, can also be described with high accuracy by the expression (18) for the capillary length $z / L_{\mathrm{ph}}>0.07$, as seen from Figure 1 . For $z / L_{\mathrm{ph}}<0.07$, the interference of higher modes leads to an irregular structure of the laser and wakefields, which determines the need for their filtering, as indicated above. In particular, for the case considered in Figure 1, we can write expressions for the damping factors of the first few modes in the form $\quad \Gamma_{n}(z)=\exp \left(-\Upsilon_{n} z / L_{\mathrm{ph}}\right) \quad$ with 


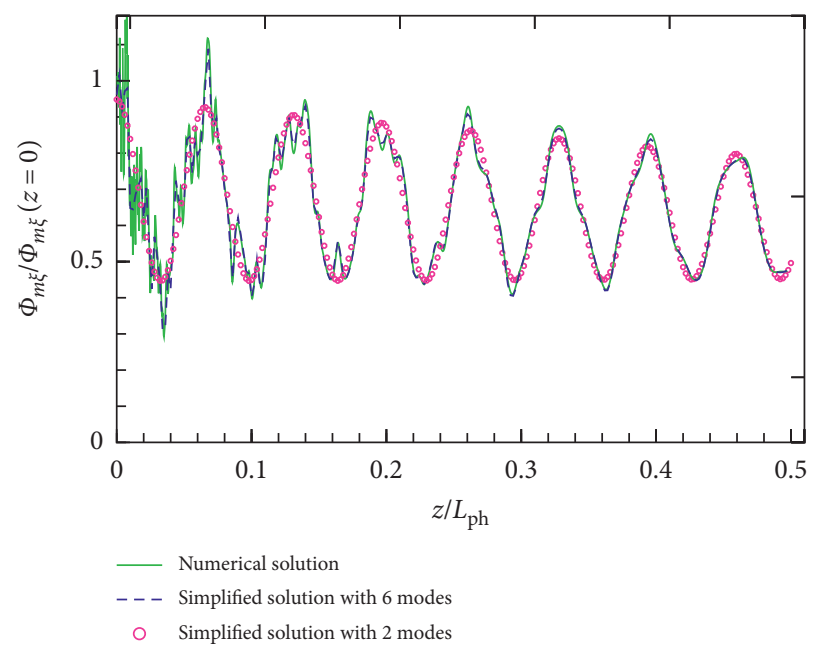

FIgURE 1: The maximum in the $\xi$ value of the wakefield potential on the capillary axis, $\phi_{m \xi}(z)$, normalized to the value $\phi_{m \xi}(z=0)$, as a function of the propagation length $z=c t$ of the laser pulse in the capillary, for the dimensionless maximum amplitude of the laser field $a_{\max }=0.5$, laser wavelength $\lambda_{0}=0.8 \mu \mathrm{m}$, plasma wave gamma factor $\gamma_{\mathrm{ph}}=80$, exponential width of the Gaussian transverse distribution of the laser field at the capillary entrance $r_{0}=50 \mu \mathrm{m}$, the full width of the laser pulse at half its maximum intensity (at $\left.z=-0\right)$, $t_{\mathrm{FWHM}}=80 \mathrm{fs}$; and a silicon capillary waveguide $\left(\varepsilon_{w}=2.25\right)$ has a radius of $R=82 \mu \mathrm{m}$ (the ratio $r_{0} / R \approx 0.61$ is close to the condition for the best laser energy input into a capillary [26]). The length $z$ along the capillary is normalized on the dephasing length $L_{\mathrm{ph}}=\lambda_{0} \gamma_{\mathrm{ph}^{3}} \approx 41 \mathrm{~cm}$. The complete numerical solution (14) (with $\tau=k_{p} z$ ) and the simplified analytical solution (18) with 2 and 6 modes are shown by different curves (see figure legend).

$\Upsilon_{1} \approx 0.202, \Upsilon_{2} \approx 1.07, \Upsilon_{3} \approx 2.62, \Upsilon_{4} \approx 4.87, \Upsilon_{5} \approx 7.80, \Upsilon_{6} \approx$ $11.43, \Upsilon_{7} \approx 15.8, \Upsilon_{8} \approx 20.8, \Upsilon_{9} \approx 26.5, \Upsilon_{10} \approx 32.9, \Upsilon_{15} \approx 75.2$. Therefore, for $z>0.1 L_{\mathrm{ph}}$, one has $\Gamma_{10}<5 \times 10^{-2}, \Gamma_{15}<10^{-3}$ that means effective filtering of high modes for such distances.
From (17), one can obtain an expression for the coefficient $\alpha$ (12) of the linear dependence of the focusing force near the capillary axis on the radius $\rho$ and, therefore, an expression for the betatron frequency $\Omega(12)$ in equation (11) for betatron oscillations. This expression can be written as

$$
\begin{aligned}
& \Omega^{2}(\tau)=\Omega_{\beta 0}^{2}(\tau) K_{g}(\tau)\left[1+\sum_{n, k>n} v_{n k}(\tau) \cos \left(\Omega_{n k} \tau\right)\right] \\
& \Omega_{\beta 0}(\tau)=\frac{2 a_{\max }}{k_{p} R} \sqrt{\frac{A}{\gamma_{e}(\tau)}}, \\
& K_{g}(\tau)=\sum_{n} C_{n}^{2} u_{0, n}^{2} \Gamma_{n}(\tau) \sin \left[\xi_{\text {inj }}+\frac{\tau}{2}\left(\frac{1}{\gamma_{\mathrm{ph}}^{2}}-\frac{1}{\gamma_{e}^{2}(\tau)}+\frac{u_{0 n}^{2}}{\mathscr{R}^{2}}\right)\right], \\
& v_{n k}(\tau)=K_{g}^{-1}(\tau) C_{n} C_{k}\left(u_{0, n}^{2}+u_{0, k}^{2}\right) \Gamma_{n k}(\tau) \sin \left[\xi_{\mathrm{inj}}+\frac{\tau}{2}\left(\frac{1}{\gamma_{\mathrm{ph}}^{2}}-\frac{1}{\gamma_{e}^{2}(\tau)}+\frac{u_{0 n}^{2}+u_{0 k}^{2}}{2 \mathscr{R}^{2}}\right)\right] .
\end{aligned}
$$

The dynamic of gamma-factor $\gamma_{e}(\tau)$ of an electron bunch can be estimated, taking into account longitudinal equation of motion, as Veisman and Andreev [18]:

$$
\gamma_{e}(\tau)=\gamma_{\text {inj }}+2 \gamma_{\mathrm{ph}}^{2} A a_{\max }^{2}\left[\sin \left(\xi_{\text {inj }}+\frac{\tau}{2}\left(\gamma_{\mathrm{ph}}^{-2}+\frac{u_{01}^{2}}{\mathscr{R}^{2}}\right)\right)-\sin \left(\xi_{\text {inj }}\right)\right] \text {, }
$$

where $\gamma_{\text {inj }}$ is the electrons gamma factor at the moment of injection.
Equation (20) shows that the squared frequency of betatron oscillations of electrons in a capillary waveguide

$$
\Omega_{\text {cap }}^{2}(\tau)=\Omega_{\beta 0}^{2}(\tau) K_{g}(\tau)
$$

is modulated in time $\tau$ with frequencies $\Omega_{n k}$ and amplitudes $v_{n k}$. In accordance with the common theory of parametric oscillator Landau and Lifschitz [30], this leads to parametric excitation of electrons betatron oscillations (the frequency $\Omega$ (20) changes with time $\tau$ due to the change in electrons gamma factor stipulated by their acceleration, due to 
attenuation of modes and dispersion of their group velocities and due to the difference between electron bunch velocity, which is close to the speed of light and the plasma wave phase velocity, manifested in dependence on $\tau$ of the factors $K_{g}$ and $v_{n k}$ in equation (20), but this change occurs adiabatically at betatron period [18], and therefore, we assume that the concept of parametric resonance is applicable for the case under consideration) provided $\Omega_{\text {cap }}(\tau)$ is close (or becomes close) to resonance frequencies (higher order resonances with lower increments are also possible for frequencies which are multiples of frequencies (23) [30]):

$$
\Omega_{R n k}=\frac{\Omega_{n k}}{2} .
$$

Maximum increments $S_{n k}$ of parametric resonances at frequencies (23) can be written, in accordance with Landau and Lifschitz [30], as

$$
S_{n k}=\frac{\nu_{n k} \Omega_{R n k}}{4} .
$$

The increments $s_{n k}$ of the betatron oscillation amplitude are nonzero in a certain interval of betatron oscillation frequencies, in the vicinity of the resonance frequencies $\Omega_{R n k}$ (23). This interval is specified by the condition that, in the following (25) expressions for $s_{n k}$ root, arguments exceed zero:

$$
s_{n k}=\sqrt{S_{n k}^{2}-\left(\Omega-\Omega_{R n k}\right)^{2}},
$$

where $\Omega_{R n k}$ are given by (23) and $S_{n k}$ are given by (24).

If $r_{\beta, 0}\left(\tau_{0}\right)$ is the amplitude of the betatron oscillations of electrons injected at $\tau=\tau_{0}$ at the point $k_{p} L_{\text {inj }}=\tau_{0}$ along the capillary length, then at a certain length $z=c t>L_{\text {inj }}$ inside the capillary, at the time $\tau=k_{p} z$, this amplitude can be estimated as $r_{\beta}(\tau)=r_{\beta, 0}\left(\tau_{0}\right) G_{n k}\left(\tau, \tau_{0}\right)$, where $G_{n k}$ is the highest resonance growth factor for a given $\gamma_{\text {inj }}$ :

$$
G_{n k}\left(\tau, \tau_{0}\right)=\exp \left(\int_{\tau_{0}}^{\tau} s_{n k}\left(\tau^{\prime}\right) \mathrm{d} \tau^{\prime}\right)
$$

The growth factors arising from the beatings of the first few modes in a matched capillary waveguide are shown in Figure 2.

From Figure 2, it is clear that beating of higher-order modes $(n, k>n, k \geq 5)$ leads to very high increments of parametric instability linear theory predicting high growth factors of parametric instability and becomes inapplicable when the amplitudes of betatron oscillations increase so strongly that they become comparable with the capillary radius $R$ for electrons with relatively low injection energies $\gamma_{\text {inj }}<150$. Instability regions are within the intervals $\gamma_{\text {inj }}<700,10^{3}<\gamma_{\text {inj }}<210^{3}, 910^{3}<\gamma_{\text {inj }}<2.510^{4}$.

Figure 3 shows the dynamics of changes in the normalized emittance $\varepsilon_{n}$ (13), the root-mean-square radius of the accelerated electron bunch $\left\langle r^{2}\right\rangle^{1 / 2}$, and the average increase in the electron energy during acceleration $\langle E\rangle-$ $E_{\text {inj }}$ (where $E_{\text {inj }}$ is the energy of the electron bunch at the moment of injection). Curves are shown for electrons with different injection energies propagating in a matched

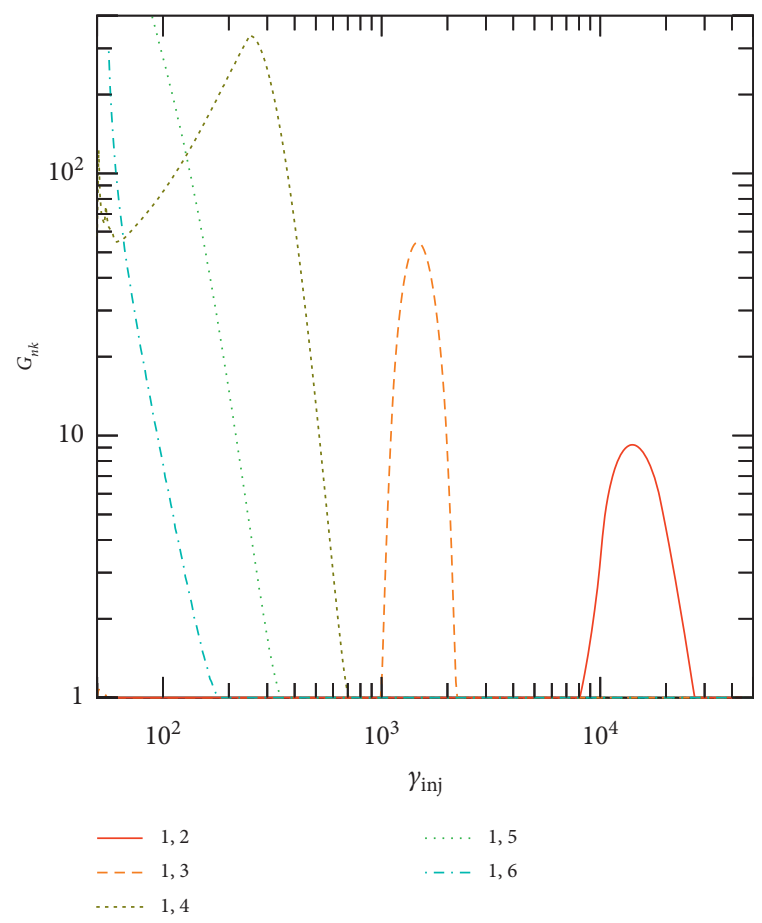

FIgURE 2: The growth factors $G_{n k}$ of betatron oscillations amplitudes as function of gamma-factors of injected electrons $\gamma_{\text {inj; }}$, calculated by formulas (20)-(25). $G_{n k}$ are estimated for a capillary waveguide with the same parameters as in Figure 1, for the duration of electron acceleration $\Delta \tau=\tau-\tau_{0}=0.2 k_{p} L_{\mathrm{ph}}$ (where $\tau_{0}=k_{p} L_{\text {inj }}$ indicates the start of acceleration; $\tau_{0}=3800 \approx 0.093 k_{p} L_{\mathrm{ph}}$ in calculations). The growth factors are due to the beating of the modes with the numbers $n, k$ indicated on the legend.

capillary waveguide. Electron dynamics in the wakefields given by the expression (14) was determined by means of numerical solution of the system of equations (1)-(4) for each electron of the bunch.

To avoid defocusing due to high (with numbers over 10) modes near the capillary entrance, the electron injection was shifted along capillary length from the capillary entrance by the value $L_{\mathrm{inj}} \approx 0.093 L_{\mathrm{ph}}$. For the selected value of $L_{\mathrm{inj}}$, modes that violate the regular structure of the wakefield near the capillary entrance are effectively filtered, while the lower modes still survive, and their beating leads to parametric excitation of betatron oscillations of electrons in the corresponding resonance regions. In particular, electrons with $\gamma_{\text {inj }}=500$ and $\gamma_{\text {inj }}=1500$ undergo parametric excitation of their betatron oscillations due to the beats of 1.4 and 1.3 modes, respectively, as follows from the results shown above in Figure 2. This excitement manifests itself in a sharp increase in the rms radius $\left\langle r^{2}\right\rangle^{1 / 2}$ and normalized emittance $\varepsilon_{n}$ (see thick and thin solid curves on Figures 3(a) and 3(b)).

On the contrary, electrons with $\gamma_{\text {inj }}=850$ and $\gamma_{\text {inj }}=7000$ are not subjected to parametric excitation of their betatron oscillations since they are outside the parametric instability regions shown in Figure 2. For these electrons, both the root-mean-square radius $\left\langle r^{2}\right\rangle^{1 / 2}$ and the normalized emittance $\varepsilon_{n}$ are bounded, respectively, by the values $k_{p}\left\langle r^{2}\right\rangle^{1 / 2}(\tau=0)=0.21$ and $\varepsilon_{n}<1 \mathrm{~mm} \cdot \mathrm{mrad}$. 


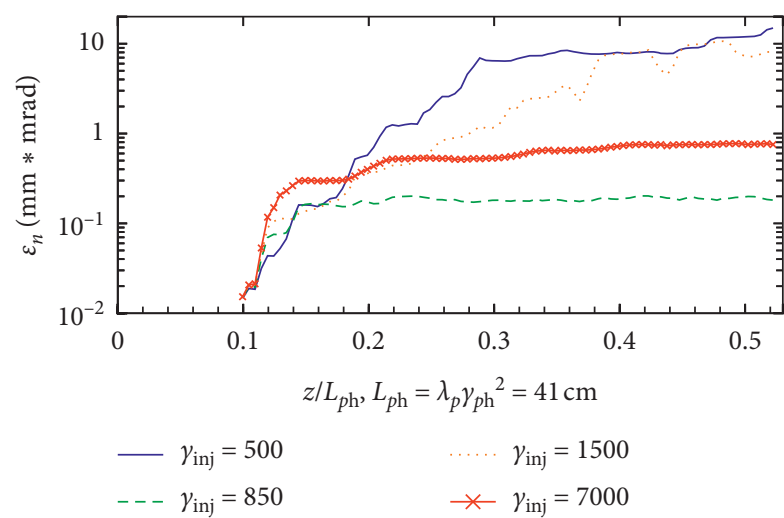

(a)

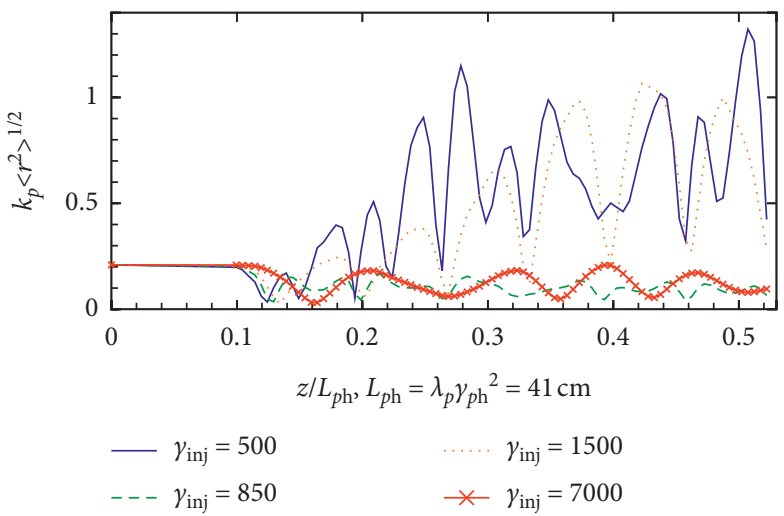

(b)

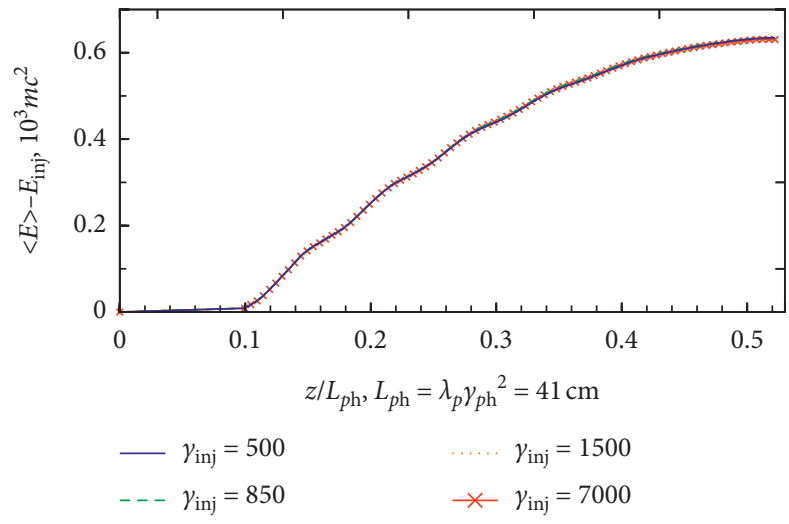

(c)

FIGURE 3: Dependencies on the acceleration length $z$ of (a) normalized emittance $\varepsilon_{n}$, (b) root-mean-square radius of the accelerated electron bunch $\left\langle r^{2}\right\rangle^{1 / 2}$, and (c) average energy gain of electrons during acceleration $\langle E\rangle-E_{\text {inj }}=m c^{2}\left(\gamma_{e}-\gamma_{\text {inj }}\right)$ for matched capillary waveguide. Curves are shown for different energies of injection of electron bunches $E_{\text {inj }}=m c^{2} \gamma_{\text {inj }}$ (see legend). Electrons in accelerated bunches have Gaussian distribution in both longitudinal and transverse directions with dimensionless characteristic sizes $k_{p} \sigma_{\xi}=0.14$ and $k_{p} \sigma_{r}=0.21$, respectively. Electron bunches are injected at the time $\tau_{0}=k_{p} L_{\text {inj }}$ at the point $k_{p} L_{\text {inj }}=0.093 k_{p} L_{\mathrm{ph}}=3800$ at the length of the capillary, with zero initial emittance. The center of the electron bunch is injected into the focusing phase of the wakefield at a distance of $\xi_{\text {inj }}=0.2$ from the point of maximum of the accelerating force. Other parameters are the same as for Figure 1.

\section{Estimation of Synchrotron Radiation}

Regular calculation of the emission spectrum of accelerated electrons requires integration over exact trajectories of electrons [31], but estimates of the intensities of the corresponding spectral lines, characteristic frequencies, and spectral widths can be made on the basis of the assumption of harmonic oscillations of electrons with constant energies and amplitudes.

In accordance with [31], the energy radiated in the direction $0 z$ (angle $\theta=0$ ) in a unit solid angle $\mathrm{d} \Theta$ in a unit frequency range $\mathrm{d} \omega$ (or in a unit energy range $\hbar \mathrm{d} \omega$ ) during $N_{\beta}=z / \lambda_{\beta}$ betatron periods (where $\lambda_{\beta}=2 \pi c / \Omega$ and $\Omega$ are the wavelength and frequency of betatron oscillations, respectively), due to synchrotron radiation of an electron, oscillating with constant amplitude $r_{\beta}$ can be written as

$$
\begin{aligned}
\frac{\mathrm{d}^{2} E_{\omega}}{\hbar \mathrm{d} \omega \mathrm{d} \Theta} & =\sum_{n} Q_{n}, \\
Q_{n} & =\frac{1}{137} \frac{4 \omega}{\omega_{n}} \frac{\gamma_{e}^{2} N_{\beta}^{2} R_{n}(\omega) F_{n}\left(\alpha_{n}\right)}{1+a_{\beta}^{2}}, \\
a_{\beta} & =\frac{\gamma_{e} \Omega r_{\beta}}{c},
\end{aligned}
$$

where

$$
R_{n}(\omega)=\left[\frac{\sin \left[\pi N_{\beta}\left(\omega / \omega_{1}-n\right)\right]}{\pi N_{\beta}\left(\omega / \omega_{1}-n\right)}\right]^{2},
$$

is the resonance function, 


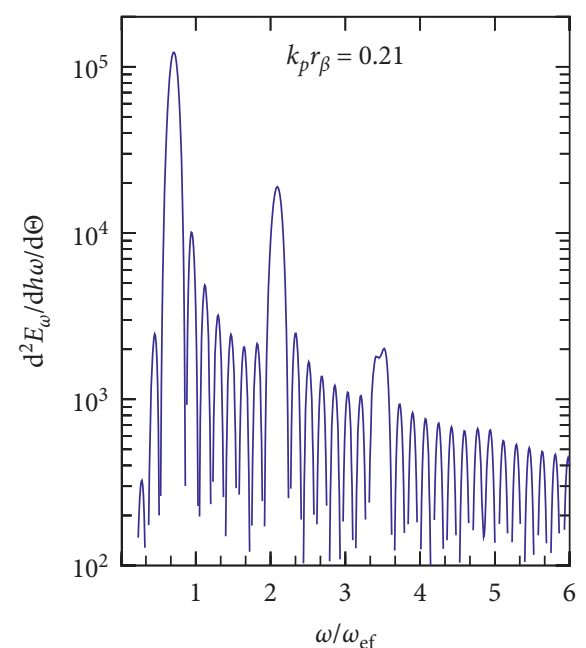

(a)

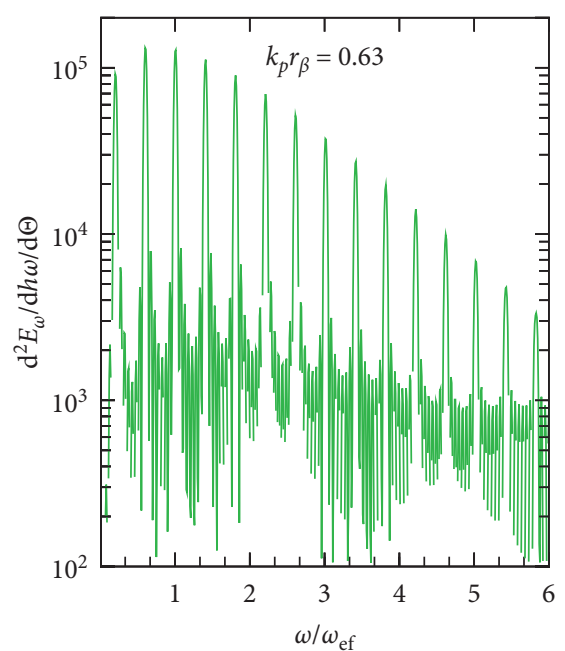

(b)

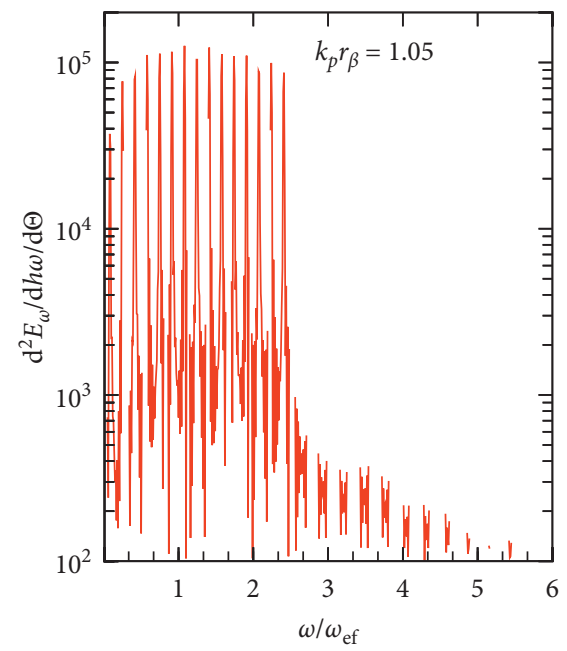

(c)

Figure 4: Energy $\mathrm{d}^{2} E_{\omega} /(\hbar \mathrm{d} \omega \mathrm{d} \Theta)$, radiated in the direction $0 z$ in a unit solid angle $\mathrm{d} \Theta$ in a unit frequency interval $\mathrm{d} \omega$, during $N_{\beta}=4$ betatron periods, for different $k_{p} r_{\beta}=0.21,0.63,1.05$ (from left to right subfigures), $\gamma_{e}=2100$, and $\Omega k_{p} L_{\mathrm{ph}}=60$. Other parameters are the same as in Figure 3. The characteristic quantum energy $\hbar \omega_{\mathrm{ef}}=260 \mathrm{eV}$ and the parameter $a_{\beta}=0.66,1.99,3.32$ for given calculations with $k_{p} r_{\beta}=0.21,0.63,1.05$, respectively.

$$
\begin{gathered}
\omega_{1}=\frac{\omega_{\mathrm{ef}}}{1+a_{\beta}^{2}}, \\
\omega_{\mathrm{ef}}=2 \gamma_{e}^{2} \Omega,
\end{gathered}
$$

and $a_{\beta}$ is given by (27); the function

$$
\begin{aligned}
F_{n}\left(\alpha_{n}\right) & =n \alpha_{n}\left[J_{(n+1) / 2}\left(\alpha_{n}\right)-J_{(n+1) / 2}\left(\alpha_{n}\right)\right]^{2}, \\
\alpha_{n} & =\frac{\omega}{\omega_{1}} \frac{a_{\beta}^{2} / 4}{1+a_{\beta}^{2} / 2},
\end{aligned}
$$

and $J_{v}$ are Bessel functions of the order $v$.

Figure 4 shows the computation of $\mathrm{d}^{2} E_{\omega} /(\hbar \mathrm{d} \omega \mathrm{d} \Theta)$ for $N_{\beta}=4$, with constant $\gamma_{e}=2100$ and for different $k_{p} r_{\beta}=$ 0.21 and $k_{p} r_{\beta}=0.63,1.05$, which estimates, respectively, the unexcited amplitude of electron oscillations and the

amplitude of oscillations excited (with growth factors $G=3$ and 5) due to parametric resonances, for the parameters shown in Figure 3.

The value of betatron frequency $\Omega$ in a capillary waveguide, calculated by expressions (20) and (22), was $1.49 \times 10^{-3}\left(\Omega k_{p} L_{\mathrm{ph}}=60.0\right)$, which corresponds to the frequency of betatron oscillations in the capillary at $\tau=$ $0.28 k_{p} L_{\mathrm{ph}}$ of an electron injected with $\gamma_{\text {inj }}=1500$ at $\tau_{0}=$ $0.093 k_{p} L_{\mathrm{ph}}$ and for all other parameters that are the same as in Figure 3. The factor $G=5$ roughly corresponds to the cases of parametric excitation of betatron oscillations, shown in Figure 3 by thick and thin solid curves. The value $\gamma_{e}=$ 2100 corresponds to the moment of acceleration $\tau \approx 0.28 k_{p} L_{\mathrm{ph}}$ for electrons injected with $\gamma_{\text {inj }}=1500$ at $\tau_{0}=$ $0.093 k_{p} L_{\mathrm{ph}}$ (thin solid curve at Figure 3 ).

A significant increase in the amplitudes of the first few harmonics of the emitted synchrotron radiation with an 
increase in $k_{p} r_{\beta}$ from 0.21 till 0.63 and 1.05 and an increase in the width of the emission spectrum are clearly seen. In the considered example of calculations with $k_{p} r_{\beta}=1.05$ (corresponding to betatron oscillations growth factor $G=5$ under conditions of Figure 3), photons are emitted in the $\mathrm{X}$-ray spectral range with photon energies $\hbar \omega \in[90 ; 650] \mathrm{eV}$ for the level of the spectral energy flux $\mathrm{d}^{2} E_{\omega} / \hbar \mathrm{d} \omega \mathrm{d} \Theta \sim 10^{5}$.

Such modifications of the synchrotron radiation spectrum with a change in the amplitude of the root-mean-square radius of an electron bunch accelerated in a capillary waveguide can serve as a diagnosis of betatron oscillations of the bunch.

\section{Conclusions}

An analysis of the parametric excitation of betatron oscillations of electrons accelerated in wakefields behind laser pulses propagating in narrow capillary waveguides with an inner radius close to the transverse dimension of the laser spot is presented. Areas (in the parameter $\gamma_{\text {inj }}$ ) of parametric instabilities due to betatron frequency modulation due to modes beats are revealed. It is shown that the dynamics of electrons during acceleration substantially depends on whether their injection energy falls into the regions of parametric instability.

It is also shown that capillary waveguides can be effectively used to accelerate electrons while maintaining a low emittance of accelerated bunches. This requires (i) excluding the influence of higher modes of electromagnetic and, as a consequence, wakefields on betatron oscillations of electrons and (ii) choosing the parameters of electron bunches and capillary waveguides in such a way to avoid regions of parametric resonances during acceleration. Parametric resonances arise from beats of modes of electromagnetic fields in capillary waveguides. Mode filtering can be performed by longitudinal displacement of the onset of acceleration (for example, by special profiling of the gas density at the capillary entrance).

On the contrary, beats of modes of the electromagnetic and wakefields in capillary waveguides or plasma channels, leading to parametric excitation of betatron oscillations of electrons, can be used in studies of sources of synchrotron radiation. Estimates have shown that an increase in the amplitude of betatron oscillations due to parametric resonances can lead to a significant increase in the width of the radiation spectrum. Thus, modifications of the synchrotron radiation spectrum with a change in the amplitude of the root-mean-square radius of an electron bunch accelerated in a guiding structure can be used to diagnose betatron oscillations of the bunch and their possible parametric excitation in this structure.

\section{Data Availability}

The data used to support the findings of this study are freely available without any restrictions.

\section{Conflicts of Interest}

The author declares that there are no conflicts of interest.

\section{Acknowledgments}

The author is grateful to Prof. N. E. Andreev for valuable discussion.

\section{References}

[1] E. Esarey, C. B. Schroeder, and W. P. Leemans, "Physics of laser-driven plasma-based electron accelerators," Reviews of Modern Physics, vol. 81, no. 3, pp. 1229-1285, 2009.

[2] C. Joshi, S. Corde, and W. B. Mori, "Perspectives on the generation of electron beams from plasma-based accelerators and their near and long term applications," Physics of Plasmas, vol. 27, no. 7, Article ID 070602, 2020.

[3] I. Y. Kostyukov and A. M. Pukhov, "Plasma-based methods for electron acceleration: current status and prospects," Physics-Uspekhi, vol. 58, no. 1, pp. 81-88, 2015.

[4] N. E. Andreev, Y. Nishida, and N. Yugami, "Propagation of short intense laser pulses in gas-filled capillaries," Physical Review E, vol. 65, Article ID 056407, 2002.

[5] B. Cros, C. Courtois, G. Matthieussent et al., "Eigenmodes for capillary tubes with dielectric walls and ultraintense laser pulse guiding," Physical Review E, vol. 65, Article ID 026405, 2002.

[6] Y. Kitagawa, Y. Sentoku, S. Akamatsu et al., "Electron acceleration in an ultraintense-laser-illuminated capillary," Physical Review Letters, vol. 92, Article ID 205002, 2004.

[7] M. Veysman, B. Cros, N. Andreev, and G. Maynard, "Theory and simulation of short intense laser pulse propagation in capillary tubes with wall ablation," Physics of Plasmas, vol. 13, no. 5, Article ID 053114, 2006.

[8] A. Curcio, D. Giulietti, and M. Petrarca, "Tuning of betatron radiation in laser-plasma accelerators via multimodal laser propagation through capillary waveguides," Physics of Plasmas, vol. 24, no. 2, Article ID 023104, 2017.

[9] M. Chen, J. Luo, F.-Y. Li, F. Liu, Z.-M. Sheng, and J. Zhang, "Tunable synchrotron-like radiation from centimeter scale plasma channels," Light: Science \& Applications, vol. 5, Article ID e16015, 2016.

[10] S. Lee, T. H. Lee, D. N. Gupta, H. S. Uhm, and H. Suk, "Enhanced betatron oscillations in laser wakefield acceleration by off-axis laser alignment to a capillary plasma waveguide," Plasma Physics and Controlled Fusion, vol. 57, no. 7, Article ID 075002, 2015.

[11] S. G. Rykovanov, C. B. Schroeder, E. Esarey, C. G. R. Geddes, and W. P. Leemans, "Plasma undulator based on laser excitation of wakefields in a plasma channel," Physical Review Letters, vol. 114, Article ID 145003, 2015.

[12] S.-W. Hwang and H.-J. Lee, "Betatron radiation of an off-axis injected electron in a laser wakefield accelerator," Journal of the Optical Society of Korea, vol. 13, no. 1, pp. 86-91, 2009.

[13] C. F. Dong, T. Z. Zhao, K. Behm et al., "High flux femtosecond $\mathrm{x}$-ray emission from the electron-hose instability in laser wakefield accelerators," Physical Review Accelerators and Beams, vol. 21, Article ID 041303, 2018.

[14] A. Døpp, B. Mahieu, A. Lifschitz et al., "Stable femtosecond $\mathrm{x}$-rays with tunable polarization from a laser-driven accelerator," Light: Science \& Applications, vol. 6, Article ID e17086, 2017.

[15] T. W. Huang, Y. F. Li, D. Z. Li et al., "Resonantly enhanced betatron hard $\mathrm{x}$-rays from ionization injected electrons in a laser plasma accelerator," Scientific Reports, vol. 6, Article ID 27633, 2016. 
[16] T. Mehrling, J. Grebenyuk, F. S. Tsung, K. Floettmann, and J. Osterhoff, "Transverse emittance growth in staged laserwakefield acceleration," Physical Review Accelerators and Beams, vol. 15, Article ID 111303, 2012.

[17] M. Reiser, Theory and Design of Charged Particle Beams, Wiley-VCH Verlag GmbH, Hoboken, NJ, USA, 2nd edition, 2007.

[18] M. E. Veisman and N. E. Andreev, "Dependence of emittance on the length of an electron bunch during laser-plasma acceleration in guiding structures," Quantum Electronics, vol. 50, no. 4, p. 392, 2020.

[19] P. San Miguel Claveria, E. Adli, L. D. Amorim et al., "Betatron radiation and emittance growth in plasma wakefield accelerators," Philosophical Transactions of the Royal Society A: Mathematical, Physical and Engineering Sciences, vol. 377, no. 2151, Article ID 20180173, 2019.

[20] N. E. Andreev and S. V. Kuznetsov, "Laser wakefield acceleration of finite charge electron bunches," IEEE Transactions on Plasma Science, vol. 36, no. 4, pp. 1765-1772, 2008.

[21] N. E. Andreev, L. M. Gorbunov, V. I. Kirsanov, K. Nakajima, and A. Ogata, "Structure of the wake field in plasma channels," Physics of Plasmas, vol. 4, no. 4, pp. 1145-1153, 1997.

[22] M. E. Veisman, S. V. Kuznetsov, and N. E. Andreev, "Growth of emittance in laser-plasma electron acceleration in guiding structures," Quantum Electronics, vol. 47, no. 3, p. 199, 2017.

[23] M. E. Veysman and N. E. Andreev, "Comparative study of laser pulses guiding in capillary waveguides and plasma channels at conditions of non-perfect focusing," Journal of Physics: Conference Series, vol. 744, Article ID 012109, 2016.

[24] N. E. Andreev, B. Cros, L. M. Gorbunov, G. Matthieussent, P. Mora, and R. R. Ramazashvili, "Laser wakefield structure in a plasma column created in capillary tubes," Physics of Plasmas, vol. 9, no. 9, pp. 3999-4009, 2002.

[25] R. Abrams, "Coupling losses in hollow waveguide laser resonators," IEEE Journal of Quantum Electronics, vol. 8, no. 11, pp. 838-843, 1972.

[26] F. Dorchies, J. R. Marquès, B. Cros et al., "Monomode guiding of $1016 \mathrm{~W} / \mathrm{cm}^{2}$ Laser pulses over 100 Rayleigh lengths in hollow capillary dielectric tubes," Physical Review Letters, vol. 82, no. 23, pp. 4655-4658, 1999.

[27] K. Lotov, "Driver channeling for laser wakefield accelerator," Particle Accelerator, vol. 63, pp. 139-146, 1999.

[28] M. Veysman, N. E. Andreev, G. Maynard, and B. Cros, "Nonsymmetric laser-pulse propagation in capillary tubes with variable radius," Physical Review E, vol. 86, Article ID 066411, 2012.

[29] N. E. Andreev and L. M. Gorbunov, "Laser-plasma acceleration of electrons," Physics-Uspekhi, vol. 42, no. 1, pp. 49-53, 1999.

[30] L. Landau and E. Lifschitz, Course of Theoretical Physics, V1, Mechanics, Elsevier, Amsterdam, Netherlands, 1982.

[31] E. Esarey, B. A. Shadwick, P. Catravas, and W. P. Leemans, "Synchrotron radiation from electron beams in plasma-focusing channels," Physical Review E, vol. 65, Article ID 056505, 2002. 